\title{
From Service Design to Innovation through Services: Emergence of a Methodological and Systemic Framework
}

\author{
Florie Bugeaud, Paul Pietyra, and Victor Liger \\ Nekoé, 16 rue Léonard de Vinci, 45000 Orléans Cedex 2, France \\ \{florie.bugeaud, paul.pietyra, victor.liger\}@nekoe.fr
}

\begin{abstract}
The current crisis conducts companies to seek new solutions for growth. In this context, the still new field of Service Design (SD) is gaining more and more attention. Easy to use and based on collaborative processes, it enables to achieve quite quick results. However the SD process is mastered by few actors inside organizations. There is a need to scale up the process to the whole organization. To achieve that goal, SD needs to undergo an industrialization process. What's more, doing SD projects is not enough to change a company. SD has to be linked to transformation strategies. This paper introduces a dedicated framework which is based on four basic components all along the Service Design process: Knowledge, Software tools, Communities and Places. The paper mainly focuses on the first two components. It will be illustrated with a complete demonstration during the session.
\end{abstract}

Keywords: Service Design, Knowledge Management, Service innovation software, service strategy, collaborative process and tool, innovation places.

\section{Introduction}

Considering the manufacturing slowdown of these last decades [1], the deep change of our economy and society towards services, and the policies that have been advocated over the world, the reindustrialization is still a challenging topic. Service appears as an interesting economic, social, emotional response. Companies seek for new sources of growth beyond the mere production of tangible goods and value of utility. They have discovered the strategic role of services and try to strengthen their culture and strategy towards this direction [2]. But they are completely disarmed against this fuzzy notion and the way of finding opportunities, developing and deploying them [3], and even more against the required global transformation.

Service Design [4] is useful to address these issues. It is a relatively new approach. Most of the Service Design process is done during collaborative workshops, with the use of tools and methods helping the co-design of the service. But they are still handmade. Capitalization, semi-automatic analysis, process acceleration, remote collaboration, indicators recovery in real time, etc. are thus not possible. Moreover launching a service (successful or not) does not mean for a company to undergo a sufficient transformation to survive or grow on a long run. Some Service Design projects enable to reach both goals (as the Gjensidige insurance company project, in 
Norway, that has succeed in shifting from a pure insurance point of view to the design of new services for people aged 20 to 30 based on a banking and insurance bundle [5]), but most of them don't.

This paper introduces a systemic framework to bring a comprehensive and equipped approach of the service innovation process to companies. To respect the paper format, we only present some screenshots but the session will be the occasion of a case study demonstration. Section 2 presents the service innovation [6] approach as a holistic and opened [7] but still handmade practice. It starts from the general Double Diamond diagram and proposes a framework that deals with the necessary construction of a long term strategy. Section 3 details two main components of this framework: Knowledge and Tools. It gives an overview of the exiting tools and presents Umagus, a dedicated software. It also introduces the two other components: Places and People. Finally, Section 4 concludes and gives some perspectives.

\section{Building a Holistic Approach for Service Innovation}

\subsection{A Service Innovation Knowledge at the Scale of the Whole Organization}

Even if is a quite general guide applicable to all design tasks, most of the academics and practitioners agree to describe the global Service Design process in the Double Diamond shape proposed by the British Design Council in 2005 [8]. It is based on 4 major phases: Discover, Define, Develop and Deliver (figure 1).

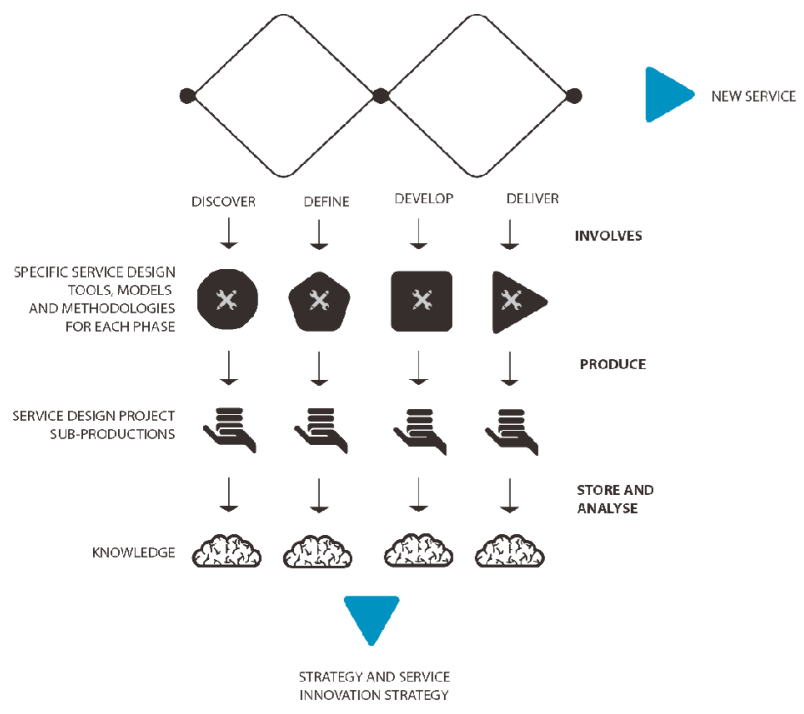

Fig. 1. The Double Diamonds shape within a global strategic reflection

Each phase allows achieving new steps in the innovation project. At the end the company should had a clear outcome: a brand new innovative service. But the Service Design process has to be kept alive after the end of the project... There are still very 
few companies having a real service innovation department, policy and/or strategy. Moreover, many valuable outcomes (knowledge, deliverables and interactions) are lost after the double-diamond process.

\subsection{A Handmade Practice That Requires a More Structured Approach}

To do Service Design [4], most of actors regularly use a common set of tools and methodologies such as personas, customer journeys, stakeholders mappings, service blueprints, prototyping (storyboards, mockups, aso.) and business model canvas. This Service Design methods and models are often used during collaborative workshops to find opportunities/ideas and/or to conceive a service. They vary from phase to phase. Sometimes the tool is an outcome that may be used directly all along the innovation and the delivery process (eg. Personas). Sometimes the tools are just a way to achieve a step (eg. stakeholders maps). But they all are rich findings and productions gathering a lot of information and knowledge that are lost or unused due to the nature of the materials (post-it, paper, etc.) and the temporary nature of the moment (fig.2).
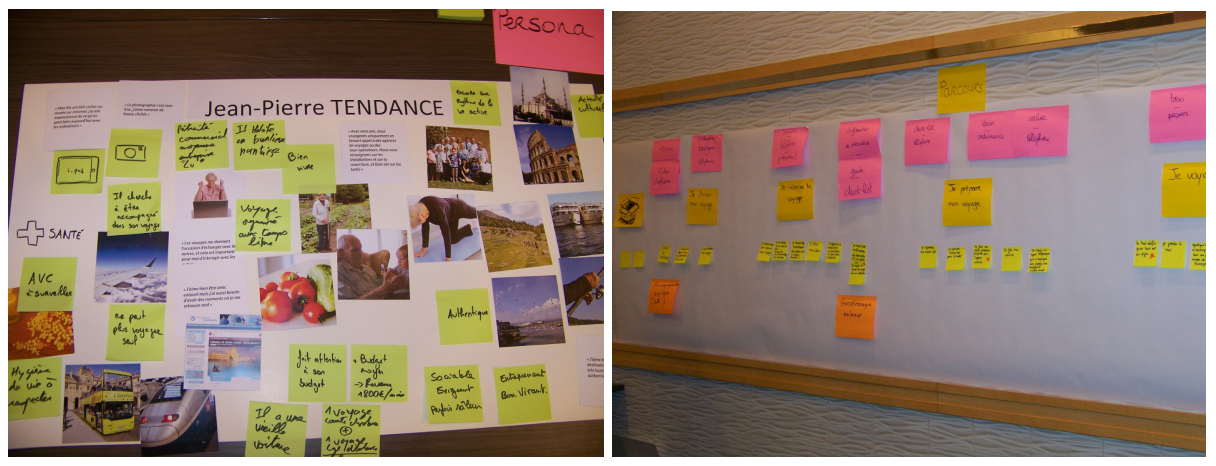

Fig. 2. Examples of handmade persona and customer journey

We argue that these findings and productions are largely underestimated on a long run, and very rarely used to build a long term strategy. That's why we propose the following framework to store, manage and analyze all service innovation projects' findings and productions to "feed" a continuous service design portfolio management linked to the global organization strategy, and to be able to achieve its transformation. Moreover, companies' service design stakeholders often are a distributed and multidisciplinary (engineers, sociologists, economists, etc.) community who has to describe and analyze complex phenomena. This leads to a lack of collaboration and consensus, and a risk for the service and product-service launched on the market.

\subsection{A Framework to Define a Transformation Process through Services}

These difficulties lead us to the assumption that there is a need for a comprehensive approach of the service innovation process and for an appropriate way of spreading a real service design culture among a company. Taking this into account we propose a 
new framework (figure 3) that will be partly developed in section 3. It aims to enrich the Double-Diamond horizontal logic (figure 1) with a vertical and systemic logic.

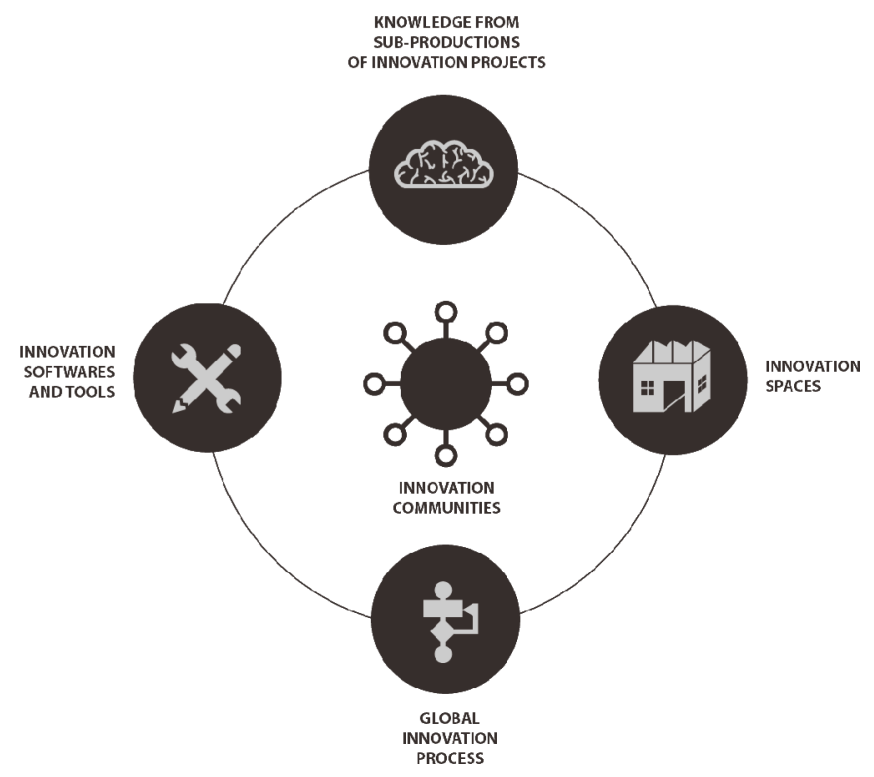

Fig. 3. Methodological framework for a global transformation through service

The systemic perspective of this framework aims to ensure the consistency between the transverse processes of innovation and capitalization, the findings and productions it generates, the tools that are used to industrialize it, the places and the communities that use them. We are developing each inter-related building block according to this systemic perspective. The global innovation process relies on a very iterative approach to design and test new services based on the users knowledge and, at a higher level of abstraction, to consider all strategic and organizational insights and constraints to implement a successful transformation. This paper focuses on two findings/productions and softwares/tools, and briefly presents the two other blocks.

\section{Using the Framework to Foster Service Innovation and Companies' Transformation}

\subsection{The Knowledge Findings and Productions of Service Design Projects as Key Elements to Identify the Strategic Constants}

The findings and productions of Service Design projects is the container and revealer of all the knowledge and data of the under construction service. In a non-exhaustive way and in relation to the Double Diamond phases, it takes the form of: 
- «discover»: a set of ethnographic «traces» such as photos, videos, verbatims, etc. ; a set of knowledge data stemming from the watchful activities ; a set of discovery deliverables such as personas, maps... ; a set of outcomes from the co-design workshops such as reports, posts-its photos...

- «define »: prototypes, tests reports, more advanced deliverables, strategical reports, design constraints, service blueprints, user experience repository....

- others phases: service specifications, touchpoints, job descriptions...

The definition, capture, treatment and reuse of these materials (cards, post-its, recordings, photos, videos, logs, etc.) within each service design project are essential to ensure the successful design and delivery of the service and the global transformation. Indeed, these elements have to allow the identification of strategical constants. They put forward insights, concepts, moments of truth, user perceptions, risks, cultural and organizational failures...revealing the need for strategical decisions, processes improvement, human resources recruitment and training, technical development, and so on. Thus service design methods and tools have to be part of a decision-aided approach that intends to bring growth and differentiation to companies.

\subsection{Existing Tool-Related Works to Support Some Service Design Phases}

Few works exist on the capture and semi-automation of required elements. Most of them propose creative methodologies and toolkits but few works really speak about a technological and complete solution. However, it is essential to capture/accumulate a lot of service design knowledge and data within a specific database to envisage the comparison and analysis of designed services or service design processes.

We can cite an Austrian initiative called myServiceFellow [9] and stemming from the results of a research project of the Management Center Innsbruck - MCI Tourism, financed by the Tyrolean science fund and the hardware kindly supported by mobilkom Austria. myServiceFellow was a mobile ethnography application for smartphone. It allowed the capture of customer journeys and their enrichment (photo, video, voice). We can also quote the well-known Swiss works on the automation of Business Model Canvas modeling/use [10] through an application available on iTunes. Another interesting project is the European project MEPSS (Methodologies for Product-Service System) that has worked on a web-based tool for the development of product-service systems. MEPSS proposed a modular approach to guide companies in the process of "developing and implementing a successful and sustainable productservice system"[11]. The Creative Design Institute of the University of Sungkyunkwan is also working on intelligent tutoring systems that aim to investigate computer-based aid to product-service systems design. This Korean team is developing a software primarily based on the service blueprint [12].

In another field than Service Design, there are also some initiatives within the SSME (Service Science Management and Engineering) community. For example SOMF (Service Oriented Modeling Framework) has been proposed by Michael Bell and implemented in EA8 of Sparx System [13]. But, even if it is dedicated to the modeling and specification of high-level service systems thanks to a specific language and a diagram of interconnected atomic and composite entities, it is still IT-oriented. 


\subsection{Umagus, a Way to Scale Up and Automate the Overall Service Design Process}

As part of a research and development project on the transformation process through services, and in order to implement the second building block of our framework (softwares and tools) which is still not completely addressed, we have worked on the conception of a specific tool and its database called Umagus (figure 4). Umagus aims to scale up and shift from the current handmade approach to an industrialized approach with a structured and semi-automated process. In its first version, it is a web-based platform to support the innovation process and keep the value of its findings and productions. Technologically speaking, it has been developed as a SaaS platform with a graph-database given the nature of the studied phenomena as heterogeneous and interconnected entities [14] and given the few constraints of this kind of database which does not require joint relationships between pre-established tables and which can store a huge amount of data.

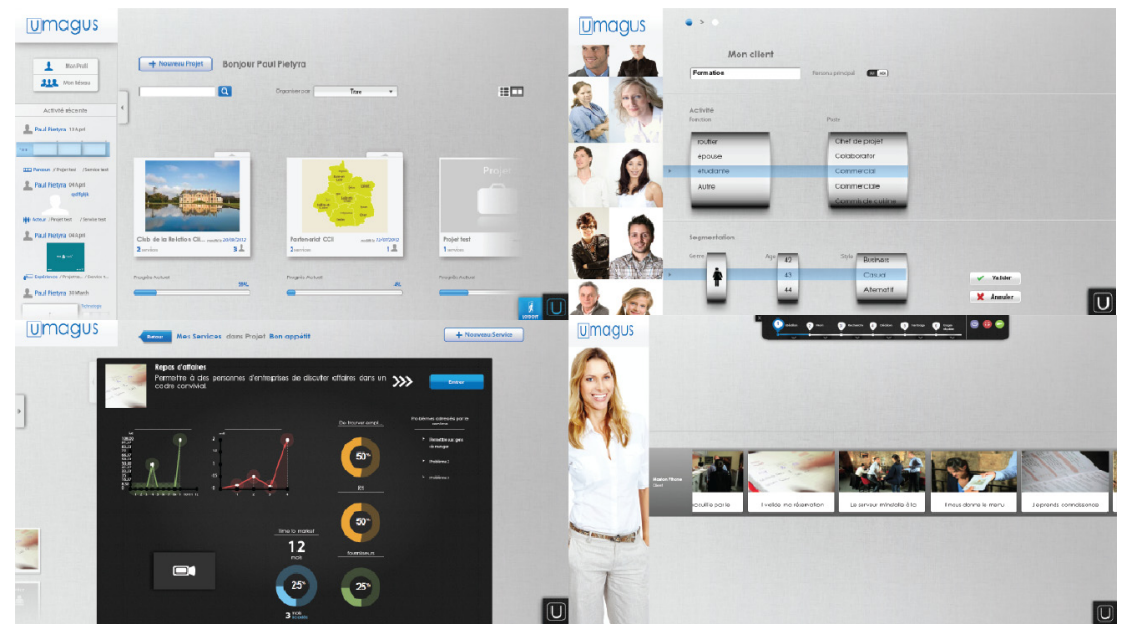

Fig. 4. Some screentshots of Umagus (projects, dashboard, persona and journey creation)

Umagus is a comprehensive "user experience-oriented" solution for services innovation and management. It enables to capture, organize and visualize "a large amount of information about the targeted company, its value chain, its activities or its users' profiles and usages" [15]. Umagus also facilitates inter-trade co-creation. Indeed, service design is natively a co-design approach. Thanks to collaborative and versioning functionalities, Umagus manages the cooperation of multiple participants in a project, allowing them to share their individual production and interact over it in a synchronous or asynchronous way, but also to think about and produce directly together. It improves the flow of data between distant stakeholders and helps them to appropriate new methods and models. It is thus a good solution to capture, store and visualize complex systems and to facilitate calculations on these systems thanks to algorithms. Key functionalities have already been implemented, tested, validated and used by some companies: service innovation projects management, heterogeneous 
data and knowledge management (user, market, aso), key methods and models integration and enrichment (persona, service journey with key stages and moments of truth, service blueprint, storyboarding...), dashboards generation (for services managers), service visualizations for different stakeholders, technology or product functionalities specifications from a service system perspective.

Umagus aims to become a key help for companies and service designers to do projects quickly and to don't lose any data and any value from the findings and productions of the service innovation process. But, to go further than the functionalities, our R\&D project now focuses on analysis and comparison of all the data that are generated by service design projects and capitalized into Umagus. We are also developing calculation methods for identifying value and innovation forms. This is a work in progress based on all the data and productions we have already captured during more than three years. It will be described in further publications.

\subsection{Innovation Places and People}

Innovation places play a more and more important role within organizations. As a short illustration, we are running a research and development project with a company in the field of aeronautics that aims to create a place and a team dedicated to innovation through services. This project has led us to translate the global service innovation process into specific spaces to meeting the needs and constraints of each phase of the double-diamond. These arrangements are built to enable the physical regular encounter between people, knowledge, tools and process. They are a real proof of the will of companies to change from service design projects to a global transformation through services.

Beyond the places, people are one of the main service innovation pillars. As for technological innovation, companies are starting to create dedicated communities for service innovation. These communities are organized around service design projects, but they are also the backbone of the organizational global change. They need specific animations and motivational tools that we are currently working on and will be the object of further publications.

\section{Conclusion and Perspectives}

Starting from the traditional Service Design process, which is based on an existing set of tools and methodologies, we have seen that companies tend more and more to develop a more comprehensive approach of service innovation, in order to achieve a global transformation of their business. Service Design needs to adapt to that trend, scaling up in terms of adoption. That's why we believe that automated solutions for service innovation and design will be more common in the future. They will be linked to global innovation solutions enabling to keep and build on the value of findings and productions of all service innovation projects. What have been done in technological innovation seems to happen also for services innovation. It is just in its early stages.

Umagus is now used during service design projects within industrial and servicial companies. We have now to pursue its use to feed the database and to integrate new kinds of analysis and calculation to make it more automated, collaborative and robust. 
Other current works concern the deployment and test of dedicated places. These perspectives and their implementation will be the objects of further publications. An oral presentation would be the occasion to concretely demonstrate our works through a simple service design case.

Finally, it seems also interesting to consider Umagus and the overall framework we conceived as possible supports for service design education since this field courses and programs are still under development and will benefit from it.

\section{References}

1. Bryson, J.R., Daniels, P.W.: The Services Duality and the Rise of the Manuservice Economy. In: Maglio, P.P., Cheryl, A.K., Spohrer, J. (eds.) Handbook of Service Science, pp. 79-104. Springer, New York (2010)

2. Edvardsson, B., Enquist, B.: Service Culture and Service Strategy - The IKEA Saga. The Service Industries Journal 22(4), 153-186 (2002)

3. Gallouj, F., Weinstein, O.: Innovation in services. Research Policy 26, 537 (1997)

4. Mager, King, O.: Methods and Processes of Service Design. Touchpoint, The Journal of Service Design 1(1), 20-29 (2009)

5. Polaine, A., Løvlie, L., Reason, B.: Service Design From Insight to Implementation. Rosenfeld Media, Paperback: 216 pages (2013)

6. Miles, I.: Innovation in Services. In: Fagerberg, J., Mowery, D.C., Nelson, R.R. (eds.) The Oxford Handbook of Innovation, Oxford Handbook Online, 656 pages (2006)

7. Chesbrough, H.: Open Service Innovation: Rethinking your business to grow and compete in a new era, 280 pages. John Wiley \& Sons (Décember 2010)

8. Design Council. The design process, London (2005), http: / /www. designcouncil.org.uk/designprocess

9. Stickdorn, M., Schneider, J.: myServiceFellow: gaining genuine customer insights. In: 1st Nordic Conference on Service Design and Service Innovation, Oslo, November 24-26 (2009)

10. Fritscher, B., Pigneur, Y.: Supporting business model modeling: A comprise between creativity and constraints. In: England, D., Palanque, P., Vanderdonckt, J., Wild, P.J. (eds.) TAMODIA 2009. LNCS, vol. 5963, pp. 28-43. Springer, Heidelberg (2010)

11. van Halen, C.J.S., Vezzoli, C., Wimmer, R.: Methodology for Product Service System innovation: How to develop clean, clever and competitive strategies in companies, 224 pages. Van Gorcum, Executive Sciences I Policy Sciences (2005)

12. Kim, Y.S., Lee, S.W., Kim, S.R., Jeong, H., Kim, J.H.: A Product-Service Systems Design Method with Integration of Product Elements and Service Elements Using Affordances. In: ServDes - 3rd Service Design and Service Innovation Conference, Helsinki, February 8-10 (2012)

13. Bell, M.: Service Oriented Modeling: Service Analysis, Design and Architecture. John Wiley and Sons, Inc., Chichester (2008)

14. Bugeaud, F.: iSamsara: An original approach to represent "servicial agencements". In: Frontiers in Service Conference, Taipei (Taiwan), July 4-7 (to be published, 2013)

15. Bugeaud, F., Pietyra, P.: Exploring data to innovate through services: a dedicated webbased solution for manufacturing and services companies. Cambridge Service Week, Cambridge (2012) 